\title{
Animal models of tracheal allotransplantation using vitrified cryopreservation
}

\author{
Hongcan Shi, MD, PhD, Hong Xu, MD, Dan Lu, MD, and Jun Wu, MD
}

\begin{abstract}
Objective: Tracheal reconstruction continues to pose a significant challenge in thoracic surgery. The study objective was to develop a novel method to eliminate the antigenicity of tracheal allografts by using vitrified cryopreservation and present the experimental results after cervical tracheal replacement in rabbits.
\end{abstract}

\begin{abstract}
Methods: Fifteen New Zealand rabbits, irrespective of gender, weighing 2.5 to $3.0 \mathrm{~kg}$, were randomly divided into 3 groups: (A), the experimental group $(n=5)$, tracheal allotransplantation after 4 weeks of vitrified cryopreservation; (B), the negative control group $(n=5)$, fresh tracheal autotransplantation; and $(C)$, the positive control group $(n=5)$, fresh tracheal segments implanted as allografts. The patency of implanted grafts, lymphocytic infiltrate, cartilage scores, and ink perfusion to evaluate revascularization were used to investigate the impact of vitrified cryopreservation on the antigenicity of tracheal grafts and vascular regeneration.
\end{abstract}

\begin{abstract}
Results: Rabbits in groups A and B all had uneventful postoperative courses with patent lumens and structural integrity, with obvious vascular regeneration and less lymphocytic infiltrate. Although in excellent condition, animals were sacrificed after a short-term follow-up of 4 weeks for further examination as scheduled. In group C, massive lymphocytic infiltrate and inflammatory cells without noticeable revascularization were observed, and rabbits died within 2 weeks after surgery for airway stenosis or severe obstruction.
\end{abstract}

Conclusion: The antigenicity of tracheal allografts was significantly decreased by using the vitrified cryopreservation method, which would be a novel alternative method to store donor trachea to make tracheal banking possible.

Immunologic rejection after tracheal allotransplantation may result in the collapse of graft structure and loss of graft function. Reducing the antigenicity of allografts and promoting revascularization are both crucial issues in current tracheal surgery. Kucera and colleagues ${ }^{1}$ pointed out that cryopreservation inhibited allogenicity while structural integrity appeared to be maintained. In the present experiment, the animal models of tracheal allotransplantation were established to study the impact of vitrified cryopreservation on the antigenicity of tracheal grafts. In addition, rejection, revascularization, and luminal patency were also evaluated after transplantation to provide experimental evidence and practical feasibility data for human tracheal transplantation.

\section{MATERIALS AND METHODS}

\section{Animals and Grouping}

Fifteen New Zealand rabbits, irrespective of gender, weighing 2.5 to 3.0 $\mathrm{kg}$, were randomly divided into 3 groups as follows: (A), the experimental

From the Department of Cardiothoracic Surgery, Clinical College, Yangzhou University, Yangzhou, Jiangsu Province, China.

Supported by the National Natural Science Foundation of China, No. 30672080; Key Medical Scientific Research Projects of Jiangsu Provincial Health Bureau (No. K200410) and Qing Lan Project of Jiangsu Province.

Received for publication Sept 1, 2008; revisions received May 1, 2009; accepted for publication May 31, 2009; available ahead of print July 27, 2009.

Address for reprints: Hongcan Shi, MD, PhD, 11 Huaihai Road, Department of Cardiothoracic Surgery, Clinical College, Yangzhou University, Yangzhou 225001,

Jiangsu Province, PR China (E-mail: shihongcan@gmail.com).

J Thorac Cardiovasc Surg 2009;138:1222-6

$0022-5223 / \$ 36.00$

Copyright (c) 2009 by The American Association for Thoracic Surgery doi:10.1016/j.jtcvs.2009.05.028 group $(\mathrm{n}=5)$, tracheal allotransplantation after 4 weeks of vitrified cryopreservation; $(B)$, the negative control group $(n=5)$, fresh tracheal autotransplantation; and $(C)$, the positive control group $(n=5)$, fresh tracheal segments implanted as allografts. All animals were provided by the Experimental Animal Center of Yangzhou University.

\section{Donor Trachea Harvesting, Cryopreservation, and Rewarming}

M199 medium solution ( $\mathrm{pH}$ 7.4) was supplemented by $3.2 \mathrm{~mol} / \mathrm{L}$ dimethyl sulfoxide, $3.2 \mathrm{~mol} / \mathrm{L}$ ethylene glycol, $0.5 \mathrm{~mol} / \mathrm{L}$ sucrose, and 2.5 $\mathrm{mol} / \mathrm{L}$ acetamide. The mixture was filtered and sterilized. After calf serum albumin $(20 \%)$, penicillin $(100 \mathrm{U} / \mathrm{mL})$, streptomycin $(100 \mu \mathrm{g} / \mathrm{mL})$, and Amphotericin B $(2.5 \mu \mathrm{g} / \mathrm{mL})$ were added, the vitrification solution was stored at $4^{\circ} \mathrm{C}$. From the fifth tracheal ring below the annular cartilage, a 5-ring tracheal segment was excised, washed with physiologic saline at $4^{\circ} \mathrm{C}$, and incubated in penicillin $(200 \mathrm{U} / \mathrm{mL})$ and streptomycin $(200 \mu \mathrm{g} /$ $\mathrm{mL}$ ) for 5 minutes. Tracheal tissue was freshly autografted, freshly allografted, or allografted after cryopreservation. Trachea allografts were cryopreserved in $50 \%, 75 \%, 85 \%$, and $95 \%$ vitrification solution $\left(4{ }^{\circ} \mathrm{C}\right)$ for 8 to 10 minutes and in $100 \%$ vitrification solution for 30 minutes, immediately followed by cryopreservation in liquid nitrogen. Four weeks later, the trachea was thawed in a thermostatic water bath at $37^{\circ} \mathrm{C}$ for 2 minutes and washed for approximately 5 minutes with thawing solution containing $1.0 \mathrm{~mol} / \mathrm{L}$ sucrose at $4^{\circ} \mathrm{C}$, followed by washing with basic solution and incubation in penicillin/streptomycin solution.

\section{Cervical Tracheal Transplantation}

Animals were anesthetized with an intramuscular injection of Sumianxin II $(0.2 \mathrm{ml} / \mathrm{kg})$ under spontaneous respiration. Sumianxin II is a compound of narcotic drugs from Changchun University of Agriculture and Animal Sciences (China) that contains dihydroetorphine hydrochloride, haloperidol, and xylidinothiazoline. The establishment of animal models in groups A, $\mathrm{B}$, and $\mathrm{C}$ followed the same operative procedures. With each rabbit in the supine position, a median incision was created. The trachea, approximately 


\section{Abbreviation and Acronym \\ $\mathrm{CSA}=$ cross-sectional area}

5 tracheal rings in length, was excised distal from the fifth tracheal ring below the annular cartilage, and the tracheal fascia was exfoliated and retained. The inner and outer diameters of the trachea were determined. Both ends of the graft were anastomosed to the native trachea. The anastomosis was begun by placing a row of interrupted sutures in the posterior membranous and then in the anterolateral cartilaginous wall. The sutures were placed at 3-mm intervals, taking approximately a 3-mm bite of trachea on each side of the tracheal margin, with the knots tied on the outside (Figure 1). After completion of the anastomosis, the strap muscles were approximated in the midline to buttress the anastomosis to increase blood supply and promote revascularization. The study protocol was approved by the ethics and animal use committee at the Yangzhou University School of Medicine.

\section{Postoperative Observations and Examinations}

Several parameters were observed and recorded after transplantation, including food intake, breath, neck incision, and body mass alterations. The causes for animal death were explored.

\section{Measurement of Grafted Tracheal Patency}

Grafted tracheal patency was represented as the patency degree of the cross-sectional area (CSA) of the most stenotic site in the graft to the third tracheal ring above the upper anastomosis in the recipient trachea: ${ }^{2} \mathrm{CSA}=$ $(\mathrm{a} / 2)(\mathrm{b} / 2)(\pi)(\mathrm{a}=$ transverse diameter; $\mathrm{b}=$ sagittal diameter $)$. The ratio of CSA before and after transplantation represented the patency degree. A patency degree of the inner cavity larger than two thirds, or stenosis smaller than one third, was regarded as well opened and without obvious stenosis. A patency degree of the inner cavity, or stenosis, ranging between one third and two thirds was regarded as generally opened but with mild stenosis. A patency degree of the inner cavity of less than one third or stenosis of more than two thirds was regarded as barely opened with severe stenosis.

\section{Measurement of the Cartilaginous Integrity and Lymphocytic Infiltrate}

Lymphocytic infiltrate and cartilaginous integrity were simultaneously assessed after transplantation. According to Nakanishi and colleagues, ${ }^{3}$ the viability of the cartilage of each transplant was graded semiquantitatively from 0 to 3, with 0 indicating severe changes (necrotic area $>70 \%$ of a microscopic visual field), 1 indicating moderate changes $(30 \%<$ necrotic area $<70 \%$ ), 2 indicating mild changes (necrotic area $<30 \%$ ), and 3 indicating the absence of any abnormalities. Lymphocytic infiltrate was scored on a 1 to 3 scale, with 1 indicating minimal infiltrate (area of infiltrate $<30 \%$ of a microscopic visual field), 2 indicating moderate infiltrate $(30 \%<$ area of infiltrate $<70 \%$ ), and 3 indicating severe infiltrate (area of infiltrate $>70 \%$ ). Each group was blindly evaluated by a pathologist.

\section{Assessment of Revascularization}

Rabbits were sacrificed, and the vascular bed was repetitively washed using 12,500 $\mathrm{U}$ heparin in $500 \mathrm{~mL}$ normal saline until light-colored or colorless liquid flew from the precaval vein. The proximal part of innominate artery was ligated and perfused with ink through an arterial cannula until ink reflux to bilateral precaval vein was observed and the vessel was fully perfused. Thereafter, the proximal part of the precaval vein was ligated. The samples were stored for 2 hours, and the tracheal graft was harvested and stored at low temperature for 24 hours, followed by fixation with $10 \%$ neutral formalin. The sections were stained with hematoxylin-eosin to evaluate revascularization.

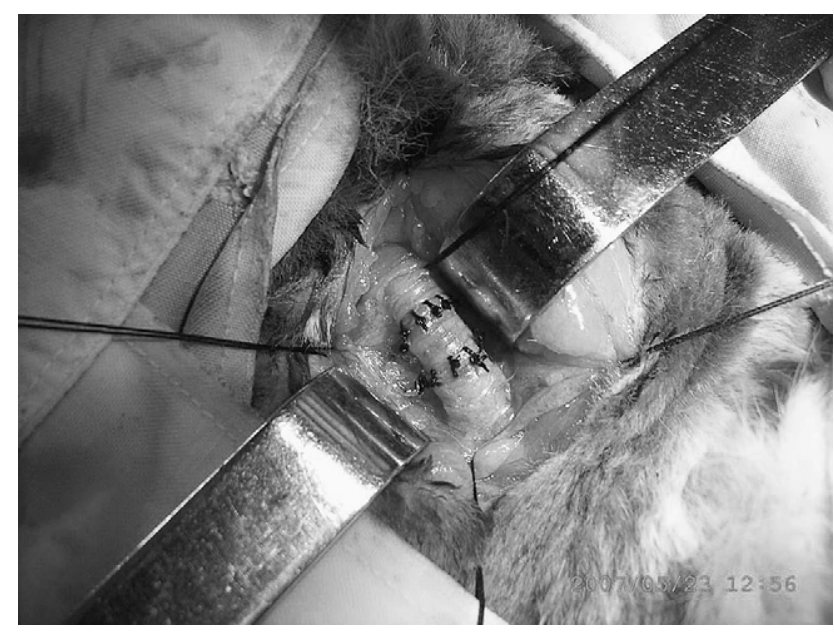

FIGURE 1. Animal model of tracheal orthotopic transplantation.

\section{Statistical Analysis}

Data are presented using mean \pm standard deviation and analyzed using SPSS 13.0 software (SPSS Inc, Chicago, IL).

\section{RESULTS}

\section{Animal Survival}

Rabbits in groups A and B all had uneventful postoperative courses without any severe complications, such as anastomotic dehiscence, abscess formation, or graft dislocation. Although in excellent condition, animals with a planned survival time of 4 weeks were euthanized as scheduled for histologic evaluation. In group C, 1 rabbit developed respiratory complications, such as tachypnea and stridor, on day 8 after operation, and then died on day 10 as a result of severe stenosis adjacent to the anastomotic site and airway obstruction with excretion. The remaining rabbits presented similar clinical features and survived no more than 2 weeks, dying of severe graft collapse or stenosis as a result of intraluminal fibrous tissue ingrowth.

\section{Tracheal Graft Patency}

Grafts in groups A and B were smooth with no signs of granulated hyperplasia. The degree of stenosis was less than $20 \%$. In contrast, local thickening and stiffness of the central canal of allografts, and secretion retention in the respiratory tract, were determined in group $\mathrm{C}$. The degree of stenosis was more than $50 \%$.

\section{Semiquantitative Cartilage and Lymphocytic Infiltrate Scores}

The semiquantitative scores of cartilage integrity and lymphocytic infiltrate are shown in Table 1 . The scores of cartilage integrity in groups $\mathrm{A}$ and $\mathrm{B}$ were significantly higher than in group $\mathrm{C}(P<.05)$, whereas there were no significant differences between groups $\mathrm{A}$ and $\mathrm{B}(P>.05)$. The lymphocytic infiltrate area in group A was markedly smaller than in 
TABLE 1. Semiquantitative cartilage and lymphocyte infiltration scores in each group

\begin{tabular}{lcc}
\hline \multicolumn{1}{c}{ Group } & Cartilage score & $\begin{array}{c}\text { Lymphocyte } \\
\text { infiltration score }\end{array}$ \\
\hline Experimental group & $2.6 \pm 0.16$ & $0.9 \pm 0.23$ \\
Negative control group & $2.9 \pm 0.10$ & $0.3 \pm 0.15$ \\
Positive control group & $2.1 \pm 0.23$ & $2.3 \pm 0.26$ \\
\hline
\end{tabular}

group $\mathrm{C}(P<.01)$, but there were no significant differences between groups $\mathrm{A}$ and $\mathrm{B}(P>.05)$.

\section{Pathologic Assessment}

In group A (Figure 2), the neoepithelium migrated from the recipient trachea near the anastomotic ends, but the degree of re-epitheliazation varied in each animal. The tracheal cartilaginous structure remained intact, with no obvious lymphocyte infiltration. Several ink-perfused small vessels were found, which grew into the cartilaginous periosteum near the cartilaginous plates.

In group B (Figure 3), the structure of autografts was intact. Many ink-perfused vessels were observed in connective tissues of the perichondrium and mucosa. In some cases, vessels penetrated the full thickness of vessel wall into the tissues below mucosa, forming vascular plexus.

In group C (Figure 4), substantial monocytes and lymphocytes infiltration were observed in the submucosa and surrounding vessels. Local necrosis or disintegration of cartilaginous plates occurred. However, no noticeable inkperfused vessels were observed in this group. Instead, some collapsed or obstructed small vessels with thickened vessel wall, surrounded by infiltrated lymphocytes.

\section{DISCUSSION}

During the past 5 decades, numerous attempts have been undertaken to deal with the challenging problems of recon-

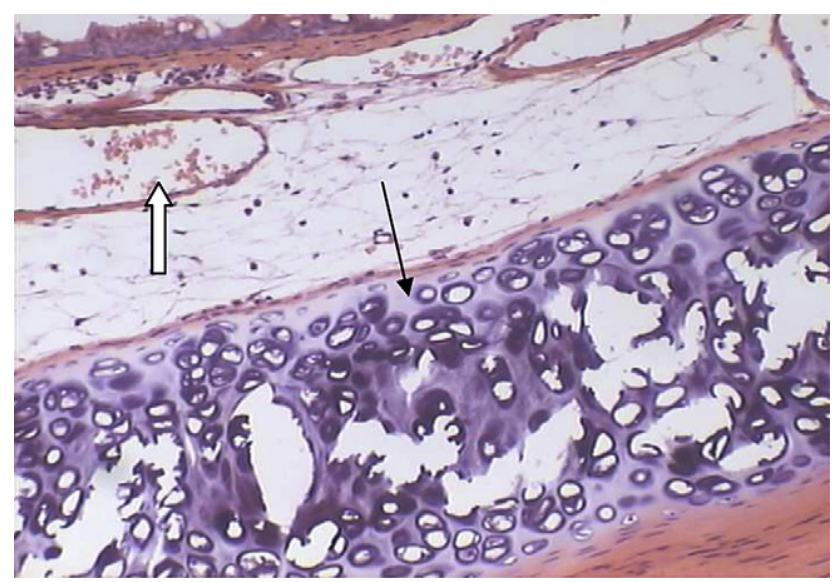

FIGURE 2. Pathologic characteristics of group A on day 28 after cryopreserved allotransplantation. Tracheal cartilaginous structure was intact (black arrow), with no obvious lymphocytes infiltration. Many ink-perfused small vessels were found (blank arrow) (original magnification, $\times 200$ ).

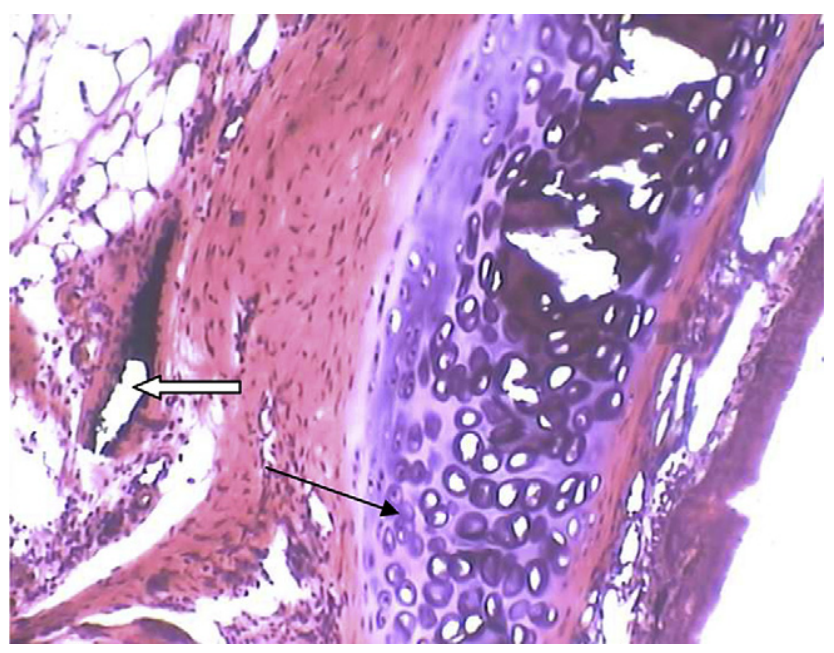

FIGURE 3. Pathologic characteristics after tracheal autotransplantation showed that the cartilaginous plates were well retained (black arrow). Ink-perfused small vessels were also found (blank arrow). From a rabbit in group B on day 28 after surgery (original magnification, $\times 200$ ).

structing long segmental tracheal defects. ${ }^{4,5}$ The spectrum of tracheal replacement ranges from autologous tissue flaps and patches to synthetic stents and prostheses to tissueengineered scaffolding. ${ }^{1,6}$ Only limited success has been achieved experimentally and clinically as a result of anastomotic stenosis, immunologic rejection, local infection, prosthetic dislocation, and material failure. ${ }^{7}$ According to Gubbels and colleagues, ${ }^{8}$ the ideal tracheal graft should (1) provide adequate structural support to maintain airway patency without the need for a luminal stent, (2) integrate into the recipient tissue to avoid the need for removal, (3) produce minimal inflammatory response to avoid scar



FIGURE 4. In one of the specimens from group $\mathrm{C}$ on day 8 , substantial monocytes and lymphocytes infiltration were observed in the submucosa and surrounding vessels, but no obvious ink-perfused vessels were observed. Local necrosis or disintegration of cartilaginous plates occurred (black arrow). No noticeable ink-perfused vessels were found (original magnification, $\times 200$ ). 
formation and stenosis, (4) rapidly mucosalize to allow for improved mucociliary transport, and (5) be readily available.

Mucous epithelium containing major histocompatibility complex II antigen may play an important role in the immunologic rejection response of the host to the tracheal graft. Several authors have reported that tracheal allograft treated by different cryopreserved methods can be used for tracheal allotransplantation. ${ }^{9-13}$ These excellent results have suggested that cryopreservation leads to the loss of the mucous epithelium from the tracheal allograft, and consequently reduces the immunogenicity. In our previous study, ${ }^{14}$ hematoxylin-eosin staining, terminal deoxynucleotidyl transferase-mediated dUTP-digoxigenin nick end-labeling staining, transmission electron microscopy, and scanning electron microscopy were conducted to assess the effectiveness of vitrified cryopreservation on rabbit trachea by comparing the vitrification process with the conventional computer-programmed slow freezing procedure. The results in our prior work showed that vitrification was less detrimental to cartilage cells and had a higher survival of chondrocytes and coverage of epithelium and cilia, which has proved the feasibility of vitrification as a storage method.

Conventional cryopreservation methods with a controlled slowing cooling rate, generated by the computer-programmed freezing device, decreased the ice-crystal formation, but ice formation was unavoidable to a certain extent, which had hazardous effects to matrix and parenchyma cells such as chondrocytes. In the vitrification method with a high-density vitrification solution combined with rapid freezing, the liquid turns into a solid and non-crystallized vitrina, which forms minimal or no ice crystals. This avoids injury to cells by minimizing damage to lipid membranes and cytoskeletal structures. ${ }^{15-17}$

In the present study, tracheal replacement of vitrified allograft was performed, and immunologic rejection and revascularization after transplantation were observed. Our study revealed that the cryopreserved tracheal allografts maintained a complete structure and a patent lumina, with less rejection-related lymphocyte infiltration compared with fresh allotransplantation. The scattered small vessels below the mucosa and periosteum indicated that vitrification reduced immunologic rejection and promoted revascularization of the grafts. This aided graft survival and luminal patency, and improved airway reconstruction. In addition, epithelium of the recipient trachea migrated irregularly and gradually from the anastomosis site to the donor trachea, but it was not confluent throughout the lumen during the observation period of 4 weeks. Nakanishi and coworkers ${ }^{3}$ previously reported that epithelial morphology was also valuable in the diagnosis of rejection after transplantation. Genden and coworkers ${ }^{18}$ demonstrated that recipient-derived tracheal epithelium migrated into the proximal donor segment within 21 days after transplantation regardless of the state of immunosuppression, and this process appeared to protect the graft lumen from obliteration. It is believed that the failure of this process to occur leads to an irreversible obliterative response. Accordingly, only animals undergoing vitrified grafting survived during the planned period up to 4 weeks, whereas those in the fresh allotransplantation group died of respiratory tract obstruction less than 2 weeks after surgery. The rejection responses after tracheal transplantation reached a peak within 2 weeks after the operation, leading to graft structure disintegration and collapse, and the loss of the airway. This experiment also demonstrated that vitrified tracheal allografts effectively reduced immunologic rejection responses and prolonged animal survival compared with the positive control group.

Genden and colleagues ${ }^{18}$ previously showed that lymphocytic infiltrate positively correlates with allograft-induced immunologic rejection. The histologic appearances in our study revealed that lymphocytic infiltrate primarily was noted in the submucosa and surrounded by small vessels near the cartilaginous periosteum. The rejection resulted in mucosal epithelium shedding and structural damage in mixed glandular organs below the mucosa. In addition, rejection induced vessel damage, or embolism, and revascularization disturbance. This further aggravated the ischemia/ hypoxia state of the grafts, resulting in cartilage collapse, disintegration, and luminal stenosis. Further studies are also needed to focus on the alleviation of damage to tissue and cells, and to maintain survival of chondrocytes.

\section{CONCLUSIONS}

Vitrified cryopreservation is a novel alternative method to store donor trachea to make tracheal banking possible, although the issues of longer follow-up and allograft patency remain to be addressed in the near future.

\section{References}

1. Kucera KA, Doss AE, Dunn SS, Clemson LA, Zwischenberger JB. Tracheal replacements: part 1. ASAIO J. 2007;53:497-505.

2. Nakanishi R, Shirakusa T, Mitsudomi T. Maximum length of tracheal autografts in dogs. J Thorac Cardiovasc Surg. 1993;106:1081-7.

3. Nakanishi R, Nagaya N, Yoshimatsu T, Hanagiri T, Yasumoto K. Optimal dose of basic fibroblast growth factor for long-segment orthotopic tracheal autografts. J Thorac Cardiovasc Surg. 1997;113:26-36.

4. Kanzaki M, Yamato M, Hatakeyama H, Kohno C, Yang J, Umemoto T, et al. Tissue engineered epithelial cell sheets for the creation of a bioartificial trachea. Tissue Eng. 2006;12:1275-83.

5. Steger V, Hampel M, Trick I, Müller M, Walles T. Clinical tracheal replacement: transplantation, bioprostheses and artificial grafts. Expert Rev Med Devices. 2008; 5:605-12.

6. Doss AE, Dunn SS, Kucera KA, Clemson LA, Zwischenberger JB. Tracheal replacements: Part 2. ASAIO J. 2007;53:631-9.

7. Shi HC, Xu ZF, Qin X, Zhao XW, Lu D. Experimental study of replacing circumferential tracheal defects with new prosthesis. Ann Thorac Surg. 2005;79: 672-7.

8. Gubbels SP, Richardson M, Trune D, Bascom DA, Wax MK. Tracheal reconstruction with porcine small intestine submucosa in a rabbit model. Otolaryngol Head Neck Surg. 2006;134:1028-35.

9. Sotres-Vega A, Villalba-Caloca J, Jasso-Victoria R, Olmos-Zúñiga JR, GaxiolaGaxiola M, Baltazares-Lipp M, et al. Cryopreserved tracheal grafts: a review of the literature. J Invest Surg. 2006;19:125-35. 
10. Hisamatsu C, Maeda K, Tanaka H, Okita Y. Transplantation of the cryopreserved tracheal allograft in growing rabbits: effect of immunosuppressant. Pediatr Surg Int. 2006;22:881-5.

11. Bakhach J, Casoli V, Guimberteau JC. The cryopreservation of composite tissues: principle, literature review and preliminary results of our own experiments. Ann Chir Plast Esthet. 2007;52:531-47.

12. Nakanishi R, Onitsuka $T$, Shigematsu $Y$, Hashimoto M, Muranaka H, Yasumoto K. The immunomodulatory effect of cryopreservation in rat tracheal allotransplantation. J Heart Lung Transplant. 2002;21:890-8.

13. Stoelben E, Harpering H, Haberstroh J, di Filippo A, Wellens E. Heterotopic transplantation of cryopreserved tracheae in a rat model. Eur J Cardiothorac Surg. 2003;23:15-20.
14. Xu H, Shi HC, Zang WF, Lu D. An experimental research on cryopreserving rabbit trachea by vitrification. Cryobiology. 2009;58:225-31.

15. Yavin S, Arav A. Measurement of essential physical properties of vitrification solutions. Theriogenology. 2007;67:81-9.

16. Guan J, Urban JP, Li ZH, Ferguson DJ, Gong CY, Cui ZF. Effects of rapid cooling on articular cartilage. Cryobiology. 2006;52:430-9.

17. Dahl SL, Chen Z, Solan AK, Brockbank KG, Niklason LE, Song YC. Feasibility of vitrification as a storage method for tissue-engineered blood vessels. Tissue Eng. 2006;12:291-300.

18. Genden EM, Iskander AJ, Bromberg JS, Mayer L. Orthotopic tracheal allografts undergo reepithelialization with recipient-derived epithelium. Arch Otolaryngol Head Neck Surg. 2003;129:118-23. 